\title{
Padrões de Indicação da Angiotomografia Coronária de Múltiplos Detectores na Prática Clínica: Experiência de um Centro de Referência
}

\author{
João Martins¹, Luis Maria Yordi¹,2, Alexandre Schaan de Quadros¹, Rogério Sarmento-Leite', \\ Carlos Gottschall', Carlos Jader Feldman ${ }^{1,2}$
}

\section{RESUMO}

Introdução: A angiotomografia coronária com múltiplos detectores está sendo progressivamente utilizada na prática clínica diária. Não existem estudos em nosso meio analisando o perfil dos pacientes submetidos a esse exame. Método: Estudo de coorte transversal, incluindo pacientes submetidos a angiotomografia coronária com múltiplos detectores em um centro de referência por indicação de seu médico assistente. Antes da realização do exame, foram avaliadas a presença de fatores de risco, a apresentação clínica da doença arterial coronária e os exames diagnósticos não-invasivos e invasivos já realizados. Os dados foram analisados com o programa SPSS 13.0. Resultados: A média de idade dos 689 pacientes incluídos foi de 63,7 anos e 52\% eram do sexo masculino. Em relação aos fatores de risco, $65 \%$ eram hipertensos, $17 \%$ eram diabéticos, 9,6\% eram fumantes ativos e $63 \%$ apresentavam história familiar de doença arterial coronária. Quanto à apresentação clínica, $49 \%$ dos pacientes eram assintomáticos, $16 \%$ apresentavam dor torácica atípica e 35\%, angina. Em relação à avaliação diagnóstica prévia, 25\% realizaram ecocardiograma e $21 \%$ realizaram cintilografia, e $37 \%$ dos pacientes não haviam realizado nenhum desses exames. Menos de $1 \%$ dos pacientes encaminhados não apresentava nenhuma dessas características, e a maioria apresentava três ou mais fatores de risco, sendo frequente a combinação dessa característica com exames diagnósticos alterados e história de revascularização prévia. Entre os pacientes assintomáticos, $98 \%$ apresentavam algum fator de risco e $27 \%$ tinham algum exame prévio positivo. Conclusão: A maioria dos pacientes encaminhados para angiotomografia coronária com múltiplos detectores

\section{ABSTRACT}

Multidetector Computed Tomographic Angiography of Coronary Arteries in Daily Clinical Practice: Experience of a Tertiary Center

Background: Multidetector computed tomographic angiography of coronary arteries has been increasingly used in the daily clinical practice. There are no studies in Brazil analyzing the profile of patients submitted to this new test. Method: Cross-sectional cohort study including patients submitted to multidetector computed tomographic angiography at a reference center and referred by their attending physician. Before angiotomographic evaluation, patients were assessed for the presence of risk factors, clinical presentation and previous results of invasive and non-invasive tests. Data were analyzed using SPSS 13.0. Results: Mean age of the 689 patients was 63.7 years and $52 \%$ were men. As to risk factors, $65 \%$ were hypertensive, $17 \%$ were diabetic, $9.6 \%$ were smokers, and $63 \%$ had family history of coronary artery disease. As to the clinical presentation of the disease, $49 \%$ were asymptomatic, $16 \%$ had atypical chest pain and $35 \%$ had angina. Twenty-five percent of the patients had echocardiography, $21 \%$ had myocardial scintigraphy and $37 \%$ had no previous tests. Less than $1 \%$ of the patients did not have any of these characteristics and most of them had three or more risk factors, frequently associated to abnormal diagnostic tests and prior myocardial revascularization. Among asymptomatic patients, $98 \%$ presented at least one of the risk factors and $27 \%$ had a previous positive test. Conclusion: Most of the patients referred for multidetector computed tomographic angiography in this

\footnotetext{
${ }^{1}$ Instituto de Cardiologia do Rio Grande do Sul - Fundação Universitária de Cardiologia (IC-FUC) - Porto Alegre, RS, Brasil.

${ }^{2}$ Serviço de Investigação Diagnóstica por Imagem (SIDI) - Porto Alegre, RS, Brasil.

Correspondência: João Martins. Instituto de Cardiologia do Rio Grande do Sul. Av. Princesa Isabel, 395 - Porto Alegre, RS, Brasil CEP 90620-001

E-mail: jmaxmartins@hotmail.com

Recebido em: 1/12/2009 • Aceito em: 26/2/2010
} 
Martins J, et al. Padrões de Indicação da Angiotomografia Coronária de Múltiplos Detectores na Prática Clínica: Experiência de um Centro de Referência. Rev Bras Cardiol Invasiva. 2010;18(1):50-4.

incluídos neste estudo apresentava fatores de risco ou exames diagnósticos prévios sugestivos de doença arterial coronária.

DESCRITORES: Doença da artéria coronariana. Angiografia coronária.

A angiotomografia coronária com múltiplos detectores tem sido proposta como um método nãoinvasivo para diagnóstico da doença arterial coronária. Utilizando-se equipamentos de tomografia axial computadorizada com no mínimo 64 detectores, é possível identificar as artérias coronárias após injeção venosa de contraste radiológico, com definição suficiente para diagnosticar anomalias congênitas, lesões parietais e obstruções nessas estruturas vasculares. ${ }^{1}$ Em estudo recente, demonstrou-se apreciável sensibilidade e especificidade dessa técnica $(85 \%$ e 95\%, respectivamente), sendo o valor preditivo negativo de $83 \%$ e o valor preditivo positivo de $91 \% .^{2}$ Mesmo com as limitações relativas impostas pelas arritmias e alta frequência cardíaca ou pelo alto escore de cálcio, ${ }^{3,4}$ a angiotomografia coronária com múltiplos detectores está sendo utilizada com maior frequência. As Diretrizes da Sociedade Brasileira de Cardiologia consideram a indicação dessa técnica no diagnóstico das anomalias coronárias como classe 1 e classe 2 para avaliação de estenose nessas artérias em pacientes com baixa probabilidade de doença arterial coronária e testes não-invasivos inconclusivos. Outras indicações, como estudo das pontes de safena, ${ }^{5}$ de stents $^{6}$ ou de pacientes com média ou mesmo alta probabilidade de estenose grave de artérias coronárias, encontram-se em fase de validação. ${ }^{7}$

Apesar da expansão do uso da angiotomografia coronária com múltiplos detectores no arsenal diagnóstico para avaliação da cardiopatia isquêmica, ainda não existem estudos analisando sua utilização em pacientes da prática clínica diária, o chamado mundo real. O objetivo do presente estudo é identificar as características dos pacientes submetidos a angiotomografia coronária com múltiplos detectores em um centro de referência em imagem diagnóstica.

\section{MÉTODOS}

No período de setembro de 2007 a janeiro de 2009 foram avaliados consecutivamente todos os pacientes encaminhados para angiotomografia coronária com múltiplos detectores em um serviço de referência de diagnóstico por imagens. Antes do exame, os pacientes foram entrevistados e os dados, registrados em um protocolo de pesquisa. Os sintomas foram definidos como dor atípica, angina estável e angina instável. Os fatores de risco foram registrados conforme relato dos pacientes: história familiar de doença cardíaca, sample had risk factors or previous diagnostic workup suggesting the presence of coronary artery disease.

KEY-WORDS: Coronary artery disease. Coronary angiography.

hipertensão arterial sistêmica, diabetes melito e tabagismo. Foram registrados os eventos isquêmicos pregressos e os procedimentos de revascularização miocárdica, bem como os exames diagnósticos não-invasivos e invasivos já realizados: eletrocardiograma, ecocardiograma, ergometria, cintilografia miocárdica e cinecoronariografia. Foi também registrado se os exames diagnósticos prévios estavam alterados ou não.

Todos os exames foram efetuados em um apareIho de tomografia computadorizada GE Light Speed VCT, 64 detectores, com pausa respiratória de 8 a 20 segundos. A modulação da dose de raios $X$ foi realizada a partir da metade do estudo, com o programa Snap Shot Pulse, que foi disponibilizado a partir desse período. Na ausência de contraindicações, eram administrados dinitrato de isossorbida $(0,5 \mathrm{mg}$, sublingual) e metoprolol (15 mg, endovenoso) nos pacientes com frequência cardíaca acima de 70 bpm. Foram utilizados $80 \mathrm{ml}$ de contraste radiológico não-iônico, injetados por via endovenosa com seringa eletrônica na velocidade de 4,5 $\mathrm{ml}$ por segundo.

Na análise estatística, as variáveis contínuas foram representadas pela média \pm desvio padrão e as variáveis categóricas, pelo percentil. A análise foi realizada com SPSS 13.0.

\section{RESULTADOS}

A análise dos 689 pacientes incluídos demonstrou que a maioria era do sexo masculino (52\%) e que a média de idade foi de 63,7 anos, conforme demonstrado na Tabela 1. Em relação aos fatores de risco, aproximadamente dois terços apresentavam hipertensão arterial e história familiar para cardiopatia isquêmica, sendo infrequentes diabetes melito (17\%) e tabagismo $(9,6 \%)$. Infarto agudo do miocárdio prévio esteve presente em $10 \%$ dos pacientes. Em relação à apresentação clínica, 49\% dos pacientes eram assintomáticos, $27 \%$ eram portadores de angina instável, 16\% apresentavam dor atípica e 8\% apresentavam angina estável.

Na Tabela 2 estão apresentados o número e o porcentual de pacientes que realizaram exames diagnósticos prévios antes do encaminhamento para angiotomografia coronária com múltiplos detectores, além do porcentual de pacientes com exames alterados. Observa-se que um terço dos pacientes não tinha realizado nenhum exame previamente, sendo a angiotomografia coronária com múltiplos detectores o pri- 
TABELA 1

Características clínicas

\begin{tabular}{lc}
\hline & $\begin{array}{c}\text { Pacientes } \\
(\mathbf{n}=\mathbf{6 8 9})\end{array}$ \\
\hline Sexo masculino (\%) & 52 \\
Média de idade (anos) & 63,7 \\
Tabagismo (\%) & 9,6 \\
Diabetes melito (\%) & 17 \\
Hipertensão arterial sistêmica (\%) & 65 \\
História familiar (\%) & 63 \\
Infarto agudo do miocárdio prévio (\%) & 10 \\
\hline $\mathrm{n}=$ número de pacientes. & \\
\hline
\end{tabular}

TABELA 2

Exames diagnósticos prévios

\begin{tabular}{lcc}
\hline & $\begin{array}{c}\text { Número de } \\
\text { pacientes }\end{array}$ & $\%$ \\
\hline Ecocardiograma & 171 & 25 \\
Com alteração & & 11 \\
Cintilografia miocárdica & 145 & 21 \\
Com alteração & & 9 \\
Teste de esforço & 168 & 25 \\
Com alteração & & 11 \\
Cateterismo cardíaco & 208 & 30 \\
Sem exames prévios & 253 & 37 \\
\hline
\end{tabular}

meiro exame diagnóstico escolhido pelo médico assistente para avaliação diagnóstica e prognóstica de doença arterial coronária. De todos os pacientes avaliados, $11 \%$ tinham ecocardiograma positivo; $9 \%$, cintilografia miocárdica positiva; e $11 \%$, teste de esforço alterado. História de intervenção percutânea foi relatada por 18\% dos pacientes, e $8 \%$ fizeram cirurgia de revascularização do miocárdio.

$\mathrm{Na}$ tentativa de identificar os fatores que contribuíram para o encaminhamento dos pacientes para a realização da angiotomografia coronária com múltiplos detectores, analisamos a presente amostra conforme a história prévia de vários fatores de risco (três ou mais), sintomas sugestivos de doença arterial coronária, exames diagnósticos prévios e história de procedimentos de revascularização do miocárdio. Também estratificamos a amostra estudada de acordo com a combinação dessas características, conforme demonstrado na Tabela 3. É importante ressaltar que menos de $1 \%$ dos pacientes não apresentava nenhuma dessas características, e a maioria apresentava três ou mais fatores de risco, e a combinação dessa característica com exames diagnósticos alterados e história de revascularização prévia foi frequente. Entre os pacientes assintomáticos 98\% apre- sentavam algum fator de risco e $27 \%$ tinham algum exame prévio positivo.

\section{DISCUSSÃO}

No presente estudo avaliamos as características clínicas, os fatores de risco, e o histórico de exames diagnósticos e revascularização do miocárdio prévia em aproximadamente 700 pacientes encaminhados para realização de angiotomografia coronária com múltiplos detectores em um serviço de referência para diagnóstico por imagens na Região Sul do Brasil. A angiotomografia coronária com múltiplos detectores é um dos mais promissores métodos diagnósticos desenvolvidos na última década, e seu padrão de utilização na prática clínica ainda não havia sido avaliado em nosso meio. Os principais resultados desta análise demonstram que a maioria dos pacientes já havia sido submetida a avaliação diagnóstica prévia, e que a angiotomografia coronária com múltiplos detectores foi utilizada como primeiro exame na investigação diagnóstica de doença arterial coronária em um terço dos casos. O porcentual de pacientes sem fatores de risco $^{8}$, sem apresentação clínica de doença arterial coronária, sem exame diagnóstico prévio e sem história de procedimento de revascularização foi mínimo (< 1\%), entretanto 50\% dos pacientes eram assintomáticos.

A característica da maioria dos pacientes examinados preenche os critérios de indicação da angiotomografia coronária com múltiplos detectores estabelecidos pela Sociedade Brasileira de Cardiologia e outras entidades, ou seja, são pacientes, no máximo, de risco intermediário..$^{9-11}$ A tomografia computadorizada, por se tratar de uma técnica que utiliza contraste radiológico e radiação ionizante, deve ter sua indicação bem estabelecida uma vez constatados os benefícios em relação ao risco e ao custo. ${ }^{12}$

Desde 1982 o estudo das artérias coronárias pela tomografia computadorizada tem apresentado impressionante progresso na qualidade das imagens obtidas. A partir de 2004, as sociedades internacionais de cardiologia, como American Heart Association, European Society of Cardiology e Sociedade Brasileira de Cardiologia, estabeleceram as diretrizes dessa técnica para a avaliação das artérias coronárias. ${ }^{9-11}$ Desde então inúmeras publicações científicas têm avaliado a importância de se estudar as artérias coronárias de maneira não-invasiva, com informações que poderiam eventualmente competir com a cinecoronariografia invasiva. A alta resolução espacial da tomografia computadorizada permite boa demonstração das artérias coronárias. Anomalias de origem e trajeto das artérias coronárias são fácilmente diagnosticadas. Essa malformação é encontrada em $1 \%$ dos pacientes e frequentemente carece de importância clínica. No entanto, existem situações em que eventos de alto risco podem ocorrer, como resultado do trajeto entre aorta e artéria pulmonar ou mesmo da origem na artéria pulmonar. Esses achados 
TABELA 3

Avaliação dos pacientes conforme a presença de fatores de risco, sintomas e procedimentos de revascularização do miocárdio prévios

\begin{tabular}{lcc}
\hline & $\begin{array}{c}\text { Pacientes } \\
(\mathbf{n = 6 8 9 )}\end{array}$ & 55 \\
\hline Fatores de risco (pelo menos 3) & 379 & 179 \\
Fatores de risco (pelo menos 3) + dor torácica & 186 & 26 \\
Fatores de risco (pelo menos 3) + exame diagnóstico prévio & 172 & 27 \\
Fator de risco + exame diagnóstico prévio com alteração & 124 & 42 \\
Fatores de risco (pelo menos 3) + dor torácica + exame diagnóstico prévio & 42 & 6,1 \\
Fatores de risco (pelo menos 3) + dor torácica + exame diagnóstico prévio + & 489 \\
procedimento de revascularização prévio & 675 & 71 \\
Fatores de risco (pelo menos 3) ou dor torácica & 4 \\
Fator de risco ou dor torácica & 98 \\
Sem fator de risco, dor torácica, exame diagnóstico prévio, & 0,6 \\
procedimento de revascularização prévio & \\
\hline
\end{tabular}

têm importância, particularmente em pessoas jovens. ${ }^{13-15}$ No entanto o grande desafio da angiotomografia de coronárias é a avaliação das lesões parietais e a detecção de redução do calibre desses vasos.

Estudos científicos têm avaliado a acurácia diagnóstica da angiotomografia com 64 detectores em pacientes com doença de coronária, demonstrando valor preditivo negativo de até $99 \%$ com a utilização da cinecoronariografia por cateter como método de aferição. Esse dado confere à tomografia computadorizada de múltiplos detectores eficiência para excluir doença coronária. ${ }^{16}$ No entanto, a sensibilidade, a especificidade e o valor preditivo positivo de $94 \%$, $83 \%$ e $64 \%$, respectivamente, sugerem limitações comparativamente com o método invasivo, como revelam, também, outros autores. ${ }^{2,17} \mathrm{O}$ estudo das características da placa de ateroma, quanto ao volume e ao conteúdo de cálcio e de tecido não-calcificado, bem como ao remodelamento, tem chamado a atenção no sentido de detectar lesões vulneráveis com a tomografia computadorizada de múltiplos detectores. A identificação desses elementos e do estágio evolutivo das lesões poderá contribuir na escolha da estratégia terapêutica não só medicamentosa como invasiva, selecionando pacientes de maior risco. ${ }^{18-20}$

Alguns pacientes avaliados neste trabalho foram previamente submetidos a procedimentos de revascularização do miocárdio. A possibilidade de reestenose intrastent tem estimulado a avaliação não-invasiva do stent pela angiotomografia coronária com múltiplos detectores, e em alguns estudos esse método poderia auxiliar na avaliação dessa complicação. Os apareIhos de 64 detectores, combinados com algoritmos de reconstrução apropriados, permitiram avaliar 88\% dos stents examinados, com resultados satisfatórios: sensibilidade de $91 \%$, especificidade de $94 \%$, valor preditivo positivo de $63 \%$ e valor preditivo negativo de $98 \%$. Stents de pequeno calibre, calcificações adjacentes e artefatos de movimento prejudicam a avaliação dos stents. ${ }^{6,20}$

O estudo de pontes de safena e de enxertos arteriais com a angiotomografia coronária com múltiplos detectores é favorecido pela menor mobilidade e pelo maior calibre dessas estruturas. Comparando com a cinecoronariografia por cateter, Meyer et al. ${ }^{5}$ estudaram 397 pacientes utilizando tomografia computadorizada com 64 detectores, encontrando 100\% de sensibilidade e $92 \%$ de especificidade. Nessa amostra, o valor preditivo positivo foi de $93 \%$ e o valor preditivo negativo, de $100 \% .^{5}$

O presente estudo demonstrou que a maioria dos pacientes encaminhados para realização desse método apresentava perfil compatível com risco intermediário para cardiopatia isquêmica, seja pela presença de sintomas, resultados alterados de exames diagnósticos prévios ou vários fatores de risco. A realização da angiotomografia coronária com múltiplos detectores pode ter contribuído para melhor conduta terapêutica, dependendo dos diagnósticos obtidos, o que será motivo de próxima análise.

\section{CONCLUSÃO}

A correta indicação do estudo das artérias coronárias por tomografia computadorizada de múltiplos detectores ainda depende de ensaios clínicos validando a técnica em pacientes assintomáticos e em pacientes com síndromes coronárias agudas. Avanços tecnológicos diminuindo o uso de contraste, reduzindo o tempo de obtenção de imagem e os níveis de radiação, bem como eliminando os artefatos provocados por arritmias de alta frequência proporcionarão avanços nesse método diagnóstico. No entanto, não há justificativa 

um Centro de Referência. Rev Bras Cardiol Invasiva. 2010;18(1):50-4.

para utilizar esse exame no rastreamento de doença coronária, e também não tem sido demonstrada "competição" entre angiotomografia coronária com múltiplos detectores e outros meios de diagnóstico, incluindo a cinecoronariografia por cateter. É provável que, no futuro, aparelhos denominados híbridos componham uma sala de diagnóstico e intervenção endovascular, utilizando os benefícios da tomografia computadorizada de múltiplos detectores e da cinecoronariografia por cateter.

\section{AGRADECIMENTO}

Agradecemos ao sr. Maurício Reche, por sua indispensável colaboração na editoração e formatação do manuscrito, e à sra. Amanda Pimienta da Silva, pela ajuda na coleta dos dados.

\section{CONFLITO DE INTERESSES}

Os autores declararam inexistência de conflito de interesses relacionado a este manuscrito.

\section{REFERÊNCIAS}

1. Niemann K, Oudkerk M, Rensing BJ, Van Ooijen P, Munne A, Van Geuns RJ, et al. Coronary angiography with multislice computed tomography. Lancet. 2001;357:599-603.

2. Miller J, Rochitte C, Dewey M, Arbab-Zadeh A, Niinuma H, Gottlieb I, et al. Diagnostic performance of coronary angiography by 64-row computed tomography. $\mathrm{N}$ Engl J Med. 2008;359:2324-36.

3. Hamon M, Biondi GG, Malagutti P, Agostoni P, Morello R, Valgimigli $M$, et al. Diagnostic performance of multislice spiral computed tomography of coronary arteries as compared with conventional invasive coronary angiography: a metaanalysis. J Am Coll Cardiol. 2006;48:1896-910.

4. Choi EK, Choi SI, Rivera JJ, Nasir K, Chang SA, Chun EJ, et al. Coronary computed tomography angiography as a screening tool for the detection of occult coronary artery disease in asymptomatic individuals. J Am Coll Cardiol. 2008; 52:357-65.

5. Meyer TS, Martinoff S, Hadamitzky M, Will A, Kastrati A, Schömig A, et al. Improved noninvasive assessment of coronary artery bypass graft with 64 slice computed tomography angiography in an unselected patient population. J Am Coll Cardiol. 2007;49:946-50.

6. Ehara M, Kawai M, Surmely JF. Diagnostic accuracy of coronary in-stent restenosis using 64-slice computed tomography comparison with invasive coronary angiography. J Am Coll Cardiol. 2007;49:951-9.

7. Goldstein JA, Gallagher MJ, O'Neill WW, Ross MA, O'Neil
BJ, Raff GL. A randomized controlled trial of multi-slice coronary computed tomography for evaluation of acute chest pain. J Am Coll Cardiol. 2007;49:863-71.

8. Tabela de fatores de risco para doença cardíaca. [cited 2010 jan 10]. Available from: http://www.americanheart.org.

9. Assessment of Coronary Artery Disease by Cardiac Computed Tomography - A Scientific Statement from the AHA Committee on Cardiovascular Imaging and Intervention, Council on Cardiovascular Radiology and Intervention, and Committee on Cardiac Imaging, Council on Clinical Cardiology. Circulation. 2006;114:1761-91.

10. Schroeder S, Achenbach S, Bengel F, Burgstahler C, Cademartiri $\mathrm{F}$, Feyter $\mathrm{P}$, et al. Cardiac computed tomography: indications, applications, limitations and training requirements. Eur Heart J. 2008;29:531-56.

11. I Diretriz de Ressonância e Tomografia Cardiovascular da Sociedade Brasileira de Cardiologia. Arq Bras Cardiol. 2006;87 Supl 3.

12. Fazel P, Peterman MA, Schussler JM. Three-year outcomes and cost analysis in patients receiving 64-slice computed tomographic coronary angiography for chest pain. Am J Cardiol. 2009;15:498-500.

13. Angelini P, Velasco JA, Flamm S. Coronary anomalies: incidence, pathophysiology and clinical relevance. Circulation. 2002; 105:2449-54.

14. Datta J, White CS, Gilkeson RC, Meyer CA, Kansal S, Jani $\mathrm{ML}$, et al. Anomalous coronary arteries in adults: depiction at multi-detector row angiography. Radiology. 2005;235:812-8.

15. Basso C, Mason B, Coroado D, Thiene G. Clinical profile of congenital coronary artery anomalies with origin from wrong aortic sinus leading to sudden death in young competitive athletes. J Am Coll Cardiol. 2000;35:1493-501.

16. Budoff M, Dowe D, Jollis J, Gitter M, Sutherland J, Halamert $\mathrm{E}$, et al. Diagnostic performance of 64-multidetector row coronary computed tomographic angiography for evaluation of coronary artery stenosis in individuals without known coronary artery disease. J Am Coll Cardiol. 2008;52:1724-32.

17. Raff G, Gallogher M, O'Neill W, Goldstein JA. Diagnostic accuracy of noninvasive coronary angiography using 64 slice spiral computed tomography. J Am Coll Cardiol. 2005; 46:552-7.

18. Pinto I. Tomografia computadorizada das artérias coronárias. Novos horizontes e desafios [editorial]. Rev Bras Cardiol Invasiva. 2009; 17:306-7.

19. Leber A, Becker A, Knez A, Ziegler F, Sirol M, Nikolaou K, et al. Accuracy of 64-slice computed tomography to classify and quantify plaque volumes in the proximal coronary system: a comparative study using intravascular ultrasound. J Am Coll Cardiol. 2006;47:672-7.

20. Sun J, Zhang A, Lu B, Yu W, Yang Y, Zhou Y, et al. Identification and quantification of coronary atherosclerotic plaques: a comparison of 64 CTMD and intravascular ultrasound. AJR Am J Roentgenol. 2008;190:748-54. 